\title{
SPLEEN RUPTURE DURING LABOR
}

\section{Camila Karsburg Bessow ${ }^{1}$, Rosi Pereira Balbinotto ${ }^{1}$, Ana Lúcia Letti Müller ${ }^{1}$, Jaqueline Neves Lubianca ${ }^{2}$}

Splenic artery aneurysm is a rare condition with a prevalence of less than $1 \%$ in the general population and is more common in women; however, it is the third most common cause of intra-abdominal aneurysms and the most common among visceral arterial aneurysms ${ }^{1}$. This condition was first described by Beussier in 1770; some of the risk factors often described are: fibrodysplasia, portal hypertension, splenomegaly, cirrhosis of the liver, liver transplant, pancreatitis, vasculitis, infectious mononucleosis, and pregnancy ${ }^{1-6}$.

Pregnancy is considered the most important risk factor for the rupture of this aneurysm, but the true prevalence of this event is unknown ${ }^{2}$. Increased splanchnic and splenic circulation during pregnancy has been indicated as one of the main factors in the development of aneurysms. The modifications in circulation induced by estrogen and progesterone during pregnancy may also contribute to weakening blood vessel walls, especially at the bifurcations. It is speculated that the greater the woman's parity the greater the risk of development and rupture of the aneurysm, due to the successive stresses to which the vessel wall is submitted ${ }^{3}$.

The importance of an adequate diagnosis and management of this condition, despite its low prevalence, is supported by the high rates of maternal and fetal mortality, 75 and $95 \%$, respectively ${ }^{3}$.

\section{CLINICAL CASE}

A 38-year-old pregnant woman with 41 weeks of gestational age and two previous vaginal deliveries without complications or known comorbidities was admitted to the obstetrical center of Hospital de Clínicas de Porto Alegre and treated according to a protocol for post-term pregnancy.

At admission, the patient had a cervical dilation of $2 \mathrm{~cm}$ and weak uterine contractions, and cardiac-fetal heart rate was normal. After using intravenous oxytocin for 2 hours, in an infusion pump at a concentration of $10 \mathrm{mU} / \mathrm{min}$, she presented a dynamic of three contractions in 10 min and was $5 \mathrm{~cm}$ dilated. Two hours later, during non-pharmacological approaches to relieve pain (bath in labor), she presented with strong abdominal pain, dyspnea, dizziness, and paleness. The medical team was called for assessment. At vaginal palpation amniorrhexis and complete cervical dilation were diagnosed, and the patient was sent to the delivery room in the expulsion stage.

In the delivery room her dyspnea, respiratory effort, and hypotension became worse. She was submitted to orotracheal intubation and invasive measurement of arterial pressure by the anesthesia team, and volemic replacement was started. The delivery was performed with a left mediolateral episiotomy and fetal extraction with Simpson's forceps. A female baby was born weighing $3705 \mathrm{~g}$ and with Apgar scores of 2/6/9 in the 1st, 3rd and 5th minutes, respectively, and sent directly to the care of the Neonatal Unit. Umbilical cord blood gas showed a fetal $\mathrm{pH}$ of 6.9. Immediately after delivery, the rapid response team (RRT) was called and the patient was sent to the intensive care unit (ICU) on mechanical ventilation. A bedside ultrasound was immediately performed (Focused Assessment with Sonography for Trauma, FAST), which showed a large amount of free fluid suggesting blood in the abdominal cavity. The hemoglobin levels at this time were $2.7 \mathrm{~g} / \mathrm{dL}$ (hemoglobin at admission $13.0 \mathrm{~g} / \mathrm{dL}$ ).
Clin Biomed Res. 2016;36(1):47-49

1 Gynecology and Obstetrics Residence Program, Hospital de Clínicas de Porto Alegre. Porto Alegre, RS, Brazil.

2 Department of Gynecology and Obstetrics, Faculdade de Medicina, Universidade Federal do Rio Grande do Sul (UFRGS). Porto Alegre, RS, Brazil.

Corresponding author: Camila Karsburg Bessow k.bessow@gmail.com Hospital de Clínicas de Porto Alegre Rua Ramiro Barcelos, 2350, sala 1125. 90035-903, Porto Alegre, RS, Brazil. 
An exploratory laparotomy was performed by the obstetrical and general surgery teams due to suspected uterine rupture or ruptured liver hematoma. The surgical finding was approximately $7 \mathrm{~L}$ of blood in the abdominal cavity and ruptured splenic hilum. Splenectomy was performed, keeping compresses in the abdominal cavity for hemostasis, and a peritoneostomy was made with a Bogota Bag. In 48 hours, the abdominal compresses were removed and the abdominal wall was closed, and no active bleeding from the splenic bed was identified. Anatomopathological examination confirmed the presence of a ruptured splenic artery aneurysm in the region of the hilum.

Forty-eight hours after closing the abdomen, leukogram results became worse, and the patient presented a fever peak and elevated lactate levels. An abdominal axial computerized tomography (CT) scan was performed, which suggested septic pelvic thrombophlebitis. Ampicillin-sulbactam and enoxaparin were prescribed and maintained for 14 days, with a good clinical and laboratory outcome. Extubation was performed 10 days after the delivery, when the patient left the ICU. She left the hospital after 33 days with clinical and surgical follow-up for post splenectomy care, including pneumococcal vaccine.

The newborn remained in the neonatal unit of the same hospital and was submitted to a therapeutic hypothermia protocol for 72 hours after birth, with a good outcome. She left the hospital after 15 days and is being followed up in the Pediatric Service, with appropriate neurological development so far.

\section{DISCUSSION}

Most cases of splenic artery aneurysm are asymptomatic, and the diagnosis is usually incidental. Only $20 \%$ of the patients present symptoms such as abdominal or thoracic pain ${ }^{4,5}$. Most of the cases are diagnosed after hemorrhagic hypovolemic shock sets in, due to ruptured aneurysm, which increases morbidity and mortality of this condition. It should be underscored that any non-traumatic symptomatic hypotension should be investigated and, in obstetrics, the differential diagnosis includes both very common conditions such as dehydration, ruptured ectopic pregnancy, abnormalities of the placenta or uterus, and uncommon ones, such as liver diseases and rupture of visceral aneurysms, mainly of the splenic type. It is estimated that $70 \%$ of the cases of ruptured splenic aneurysm are initially diagnosed as uterine rupture ${ }^{4}$.

In 2014, Kazaryan et al. published the report of two cases of splenic artery rupture in the absence of trauma in young women who presented to the emergency room with hemorrhagic shock, a 21-year-old non-pregnant patient and a 28-year-old primigravida with 26 weeks of gestational age. In accordance with the hospital routine, the trauma alarm was activated and the patients received prompt care and diagnosis, including ultrasound, which found a massive amount of intra-abdominal fluid. The patients were sent to surgery within 30 and 60 minutes, respectively, after arriving in the emergency room ${ }^{6}$.

Aiming to improve the differential diagnosis of hypovolemic shock in women of reproductive age, several models of protocols have already been described, including the use of advanced trauma life support (ATLS) and bedside ultrasound (FAST). The principles of ATLS developed since the mid-70s by the American College of Surgeons are a reliable tool for the initial management of trauma patients ${ }^{4,7}$. However, the trauma alarm, which consists of immediately triggering care by health professionals trained for emergency situations, can also be used at admission of all critically ill patients, even in the absence of trauma. Hemorrhagic shock most frequently occurs as a consequence of traumatic injury. Severe spontaneous intra-abdominal bleeding is very rare in the absence of coagulopathy ${ }^{8}$. Obstetrical patients with acute massive bleeding often raise diagnostic hypotheses that have an obstetrical cause, which may delay correct diagnosis. This underscores the need to include the principles of ATLS mandatorily in the training of all doctors. Our case also confirms the essential role of the surgeon in the RRT and in the assessment of emergency patients seen under the ATLS principles.

Bedside ultrasound is a useful tool in unstable patients who cannot be moved to perform more complex exams (CT scan, resonance or arteriography) and helps in diagnosing obstetrical cases of hypovolemic shock, placental abruption, uterine rupture, and of non-obstetrical causes ${ }^{4}$.

Ruptured aneurysm must be considered among the causes of hypovolemic shock in pregnancy, and early detection can reduce the morbidity and mortality of this event. The fast diagnosis of hemodynamic instability and hemoperitoneum with fast access to the operating theater, use of blood products, and the presence of a vascular surgeon during exploratory laparotomy were essential measures for an appropriate maternal and fetal outcome in this case. 


\section{REFERENCES}

1. Abdulrahman A, Shabkah A, Hassanain M, Aljiffry M. Ruptured spontaneous splenic artery aneurysm: A case report and review of the literature. Int J Surg Case Rep. 2014;5(10):754-7.

2. Nanez L, Knowless M, Modrall JG, Valentine RJ. Ruptured splenic artery aneurysms are exceedingly rare in pregnant women. $J$ Vasc Surg. 2014;60(6):1520-3.

3. Fong Ha J, Phillips M, Faulkner K. Splenic artery aneurysm rupture in pregnancy. Eur J Obstet Gynecol Reprod Biol. 2010;146(2):133-7.
4. Jackson HT, Diaconu SC, Maluso PJ, Abell B, Lee J. Ruptured splenic artery aneurysms and the use of an adapted fast protocol in reproductive age women with hemodynamic collapse: case series. Case Rep Emerg Med. 2014;2014:454923-1-454923-5.

5. Holdsworth RJ, Gunn A. Ruptured splenic artery aneurysm in pregnancy: a review. Br J Obstet Gynaecol. 1992;99(7):595-7.

6. Kazaryan AM, Wiborg J, Hauss K, Anundsen TK, Flemmen OJ, Holm $T E$, et al. Spontaneous non-traumatic massive intraabdominal spleen bleeding in young females: Importance of ATLS principles and trauma alarm. Am J Case Rep. 2014;15:189-93.

7. American College of Surgeons Committee on Trauma. Advanced trauma life support for doctors: ATLS student course manual. 8th ed. Chicago: American College of Surgeons; 2008 [cited 2015 Oct 04]. Available from: http://pt.slideshare.net/ urgenciasucc/atls-advanced-traumalife-support-8th-ed.

8. Spahn DR, Bouillon B, Cerny V, Coats TJ, Duranteau J, Fernández-Mondejár $\mathrm{E}$, et al. Management of bleeding and coagulopathy following major trauma: an updated European guideline. Crit Care. 2013;17(2):R76.

Received: Jan 11, 2016 Accepted: Feb 13, 2016 